\title{
AI BASED EXPERT SYSTEM TO AID PATIENTS WITH DEPRESSION DISORDER
}

\author{
Supriya Thakur ${ }^{1}, \mathrm{Saba}^{2}, \mathrm{Neha}^{3}$ Goliwar $^{4}$ and Dr. Nitin Pise ${ }^{5}$
}

\begin{abstract}
Depression is a common mental disorder affecting a close to 350 million of the world population according to World Health Organization (WHO). While the diagnosis of Depression is difficult due to the absence of unique pathological/physical indicators, the recovery is also complex and requires a guided approach. Currently available Online Support Forums do not provide a comprehensive connect to a Depression patient, but serve only as a repository of study material. This paper proposes an Artificial Intelligence based expert system (IGain) to help Depression patients diagnose this medical condition and also provide an interactive guidance on means to alleviate Depression symptoms. IGain can supplement the role of a doctor by providing an integrated analysis of the patient's mental health, personality and preferences, to provide suitable guidance to the patients in terms of stress relief, recreation and confidence boosting.
\end{abstract}

Keywords - Depression, Expert System, Prolog, Depression Support, Artificial Intelligence

\section{INTRODUCTION}

As per World Health Organization (WHO), 'Depression' is a common mental disorder, characterized by sadness, loss of interest or pleasure, feelings of guilt or low self-worth, disturbed sleep or appetite, feelings of tiredness, and poor concentration[1]. It affects approximately 350 Million people worldwide[2]. Various factors including but not limited to physical, mental, social, biological causes, may lead to Depression. Depression may manifest in one of the many types such as Major Depression, Bipolar or Seasonal Depression, to name a few.

Depression diagnosis is not easy due to the fact that there are no direct physical and /or pathological indicators. A lot depends on the question-answer type diagnosis by the doctor. While there are multiple channels (like support forums, counselors, guide websites, etc.) which provide online support for patients in terms of guidelines to identify depression symptoms and look for possible remedies, there may be a gap as far as the actual applicability of the suggested procedures to actual patients situation is concerned due to limited patient information being used and the decision being left to the patient or user.

We intend to propose an Expert System (IGain) based on Artificial Intelligence with a Prolog rules engine to help patients identify and deal with depression condition. This intelligent system will guide the patient to follow steps which can potentially help in alleviating signs and effect of depression. The paper also proposes how Bayesian networks can be used to predict the future state of depression and provide early warning signals.

\footnotetext{
${ }^{1}$ Department of Computer Engineering, Maharashtra Institute of Technology, Pune, Maharashtra, India

${ }^{2}$ Department of Computer Engineering, Maharashtra Institute of Technology, Pune, Maharashtra, India

${ }^{3}$ Department of Computer Engineering, Maharashtra Institute of Technology, Pune, Maharashtra, India

${ }^{4}$ Department of Computer Engineering, Maharashtra Institute of Technology, Pune, Maharashtra, India

${ }^{5}$ Department of Computer Engineering, Maharashtra Institute of Technology, Pune, Maharashtra, India
} 


\section{CURRENT OPTIONS FOR DEPRESSION TREATMENT AND I-GAIN}

\section{A. Traditional Medical Diagnosis and Treatmen}

The preferable treatment options consist of basic psychosocial support combined with antidepressant medication or psychotherapy, such as cognitive behavior therapy, interpersonal psychotherapy or problemsolving treatment. Antidepressant medications and brief, structured forms of psychotherapy are effective. Antidepressants can be a very effective form of treatment for moderate-severe depression but are not the first line of treatment for cases of mild or sub-threshold depression[3].

\section{B. $\quad$ Self Help and Online Depression Analysis and Guidance}

As an adjunct to care by specialists or in primary health care, self-help is an important approach to help people with depression. Innovative approaches involving self-help books or internet-based self-help programs have been shown to help reduce or treat depression in numerous studies in Western countries [3].

\section{Merits of 'I-GAIN'}

The 'IGain' system is meant to provide an intelligent 'Self Help' Expert Solution for Depression patients. While most available internet-based or self help options provide all possible options for the user to choose from, I-Gain will customize the suggested solution based on user profile or user's current situation and level of Depression. I-Gain will also have the potential to predict any warning signs of increasing Depression with a feature to inform the patient's, doctor, family, etc.

\section{SYSTEM OVERVIEW}

The 'IGain' will be a web based system, accessible on computers as well as mobile devices. To make a holistic analysis, we propose to provide explicit and implicit inputs to the system to detect Depression disorder.

\section{A. $\quad$ Functional Requirements}

The 'IGain' system is meant to provide an intelligent 'Self Help' Expert Solution for Depression patients. While

\section{1) User Types}

There will be three types of users of this system

1. Regular user (Patients or Potential Patients) - Type 1

2. Doctors / Counselors - Type 2

3. Buddies (Support Groups, People willing to help or share experience) - Type 3

\section{2) User Data Input}

Every type of user will create a user profile to store basic information. For Type 1 user, additional information which may include their hobbies, health inputs, habits, routine, preferences such as diet, doctor, entertainment, etc. will be input. These inputs will be stored in the PROLOG Knowledge Base and will directly be used to derive the support provided to the patient by IGain App.

For Type 2 and Type 3 users, system will input and store area of expertise, location, availability details, etc.

All users can update their IGain data, at any frequency, in any number of interactions.

3) One-time Inputs

The Prolog Knowledge base is initially fed with following data vetted by appropriate medical personnel: 
1. Information related to Types and Levels of Depression, Symptoms of Depression, etc.

2. Conditions to identify possibility of Depression from Symptoms (Direct Symptoms, Keyword based, etc.)

3. Base data on types and courses of treatment for various levels. This information can be used to suggest possible treatments for a patient, on case to case basis.

This knowledge base will be augmented from time to time with new information or updates to existing information with inputs from appropriate medical personnel.

\section{4) Help Options}

Since making the patient open up socially is an important aspect of Depression Disorder treatments, the IGain System shall perform an analysis of the user's current state and preferences, and suggest one or more of the help options available. These will also be tracked by the system as a part of the user case history. The available options can be one or more from the below indicative list.

Table 1: Help Options 1

\begin{tabular}{|c|l|}
\hline Connect to social circles & Recreation/Mind Activities \\
\hline Facebook, Suggest Friends & Foods \\
\hline $\begin{array}{c}\text { Specific circles in locality (e.g. } \\
\text { Laughing Club) }\end{array}$ & Books \\
\hline Friends & Movies, Television \\
\hline $\begin{array}{c}\text { Share Success Stories from outside } \\
\text { world/other users of IGain }\end{array}$ & Photos, Album \\
\hline Games & Television \\
\hline & Internet \\
\hline
\end{tabular}

Table 2: Help Options 2

\begin{tabular}{|l|l|}
\hline Immediate Help & Red Alert \\
\hline $\begin{array}{l}\text { Helplines, phone \#s and } \\
\text { Support Groups / Counselor }\end{array}$ & Inform Near \& Dear Ones \\
\hline $\begin{array}{l}\text { Connect to Doc (fees , } \\
\text { location, etc) }\end{array}$ & SMS to Doctor \\
\hline Medical Stores & Email to kin, trusted circle \\
\hline & Automated SMS to family \\
\hline
\end{tabular}

\section{5) Other Miscellaneous Features}

An actions tracker shall track the recommendations and successes (in terms or the user accepting the recommendation). A feature to report the same to Patient's Circle (Doctor/ Family/ Kin) shall be provided.

A reporting feature to provide Trend Analysis, Summary of Actions over a period of time will be provided. A feedback and recommendation feature will be included.

\section{B. Built in Artificial Intelligence}

The 'IGain' system is meant to provide an intelligent 'Self Help' Expert Solution for Depression patients. While 
The core strength of the IGain Expert System is its ability to derive the best possible support options for a Depression Disorder patient (or potential patient) using not just the basic Depression Condition Diagnosis Rules but also by considering the patients profile, available support options in patient's vicinity and the Patient's Circle.

\section{1) Prolog Module}

IGain will implement a Prolog Engine, which will be used to analyze the combination of inputs from a Knowledge base - which has both static and dynamic Depression diagnosis rules and the user case history - which stores user preferences and till date action history.

This Prolog Engine which will take three types of input - Explicit Rules, User State Inputs and Implicit Inputs

- Explicit Rule Database - These rules will be pre-fed into the Prolog knowledge base with inputs from appropriate medical personnel and shall be updated from time to time. These will contain the set of fixed conditions to be verified for existence of Depression symptoms and to ascertain the level and type of Depression Disorder existing in the user.

- User State Inputs - These input parameters will be derived from user profile, users' day-to-day interaction with the expert system, user data analysis and available help options based on user's profile and preferences.

- Implicit Inputs - These inputs will be taken via one or more of the smart-input mechanisms described later in this paper.

The Rule Engine will

- Identify if Depression conditions exists

- Identify type of depression

- Identify level of depression (based on direct input by user, and keyword search)

- Store user preferences and suggest suitable Help Option(s).

2) Optimal Help Option Search

Heuristic based search for finding nearby resources (Doc, Friends, Shops, etc). Integration with standard map tools such as GoogleMaps can be explored. Finding available /connected kin to support the patient or to inform about patient's health will be implemented using a tree hierarchy.

\section{3) Bayesian Network (Future State Prediction)}

IGain Expert system aims to enhance the predictability of future state of a Depression Disorder patient, by using probabilistic models. There have been many successful applications of Bayesian Network based models in medical and scientific fields.

Bayesian networks can be used to represent the available patient and diagnostic information as a graphical model. For example, if a patient's case history shows a decline in the number of helpline calls accompanied with an increase in positive activities tracked by IGain, this may be probabilistically interpreted as a decrease in depression level. By constructing a probabilistic model, IGain Expert System will use patient's data to analyze the possible future state of Depression or possible end result of the current condition for various corrective courses.

\section{SMART INPUT OPTIONS}

Various smart input options are being considered for IGain. This would involve integration with devices which measure various physical parameters such as heart-rate, blood pressure, eye movements, etc. which may be correlated with various types or levels of Depression. 
One of the options is to monitor heart rate of Type 1 user, to estimate depression scale. EmWave by HeartMath is using the heart-rate analysis by photoplethysmography (PPG) for a stress monitor. It enables convenient implementation of circuit with the chip directly on a cloth. Fabric electrodes are used to acquire required physiological signals during daily life[8].

Eye movement analysis for depression detection may also be considered as a smart input option. [9] Integration with one or more Health and Fitness Trackers such as Fitbit, Apple Watch, and Garmin can be considered, based on the applicability of parameters measured by these trackers as indicators of Depression level.

\section{V.SYSTEM ARCHITECHTURE}

The proposed IGain architecture consists of a three tier client server model consisting of front end, business logic and the database. The database used will be Microsoft SQL which would help in storing user data and other entity data. The Prolog Rule Engine will communicate with a Java User interface to obtain user inputs and other implicit indicators for analysis. The Rule engine will perform a complete analysis of user's explicit inputs and input obtained via smart sensors, relate the same to user profile and preferences, to provide a customized solution for each patient.

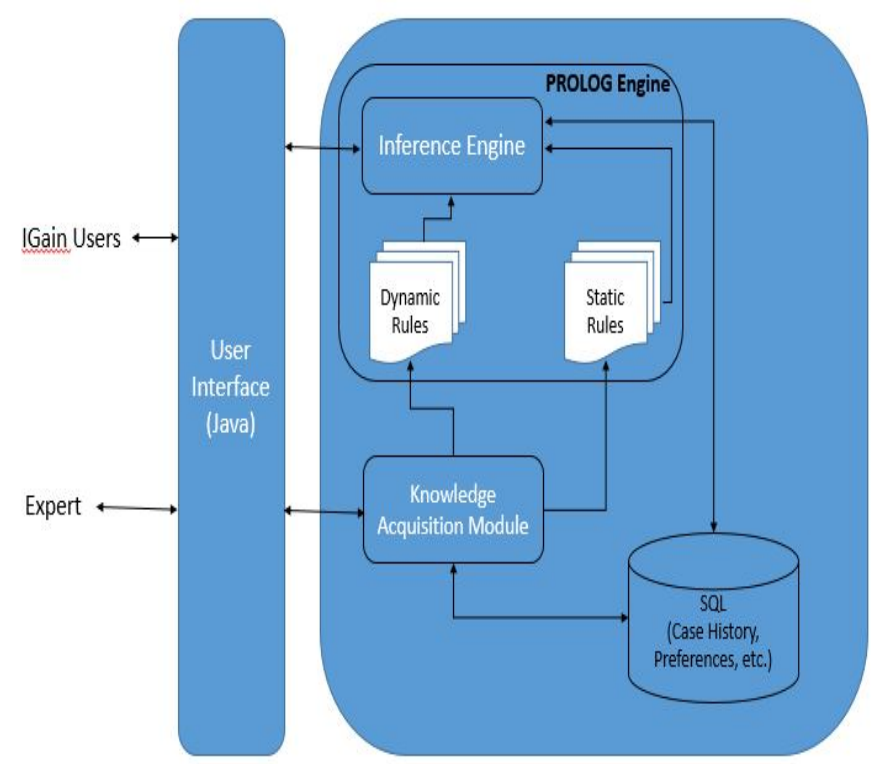

Figure 1: Proposed IGain System Architecture

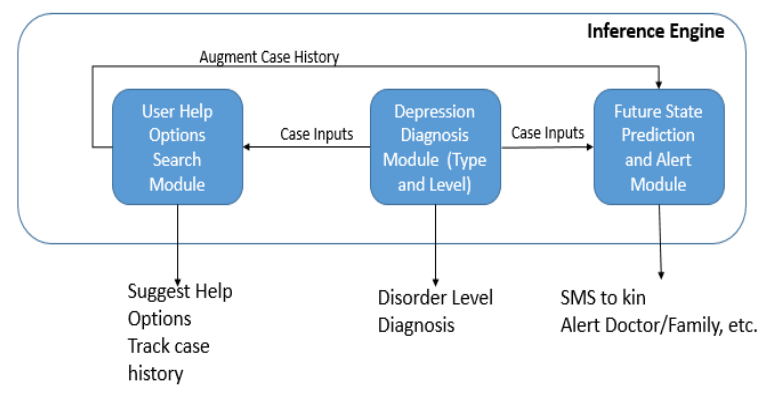

Figure 2: IGain Inference Engine 


\section{A. $\quad$ Explicit User Inputs Analysis}

Once the user profile is created, user inputs will be obtained via an interactive interface to gauge the current situation of the Type 1 user. The queries to the user will aim to find out the mental state, improvement from past interaction and any change in preferences. These will be fed to the rules engine.

\section{B. Smart Input Analysis}

One or more smart inputs suggested earlier in this paper will also feed into the Prolog Engine, so that the engine can predict a more accurate solution for the user.

\section{CONCLUSION}

An integrated expert system such as IGain, will enable online tracking of Depression Disorder patient's progress by the doctors or family and at the same time provide support and suggestions to the patient in terms of relevant guidance, tracking progress, predicting potential danger, etc. Integration with Fitness Trackers or body monitors, to enable real time tracking of body conditions to predict potential red-alert situations for Depression Disorder patients can be explored

\section{REFERENCES}

[1] "Depression" Health Topics, World Health Organization (http://www.who.int/topics/depression/en/)

[2] "Depression Fact Sheet" , Media Centre, World Health Organization, (http://www.who.int/mediacentre/factsheets/fs369/en/), Reviewed April 2016

[3] "Depression - A Global Public Health Concern" Marina Marcus, M. Taghi Yasamy, Mark van Ommeren, and Dan Chisholm, Shekhar Saxena, WHO Department of Mental Health and Substance Abuse, World Federation of Mental Health

[4] Andrews G, Cuijpers P, Craske MG, McEvoy P, Titov N. Computer therapy for the anxiety and depressive disorders is effective, acceptable and practical health care: a meta-analysis. PLoS One. 2010 Oct 13;5(10):e13196

[5] Babič, František, Adrián Jančuš, and Katarína Melišová. "Customized web-based system for elderly people using elements of artificial intelligence." Computer Science and Information Systems (FedCSIS), 2016 Federated Conference on. IEEE, 2016.

[6] Lee, Eun-Mi, and Kang-Hee Lee. "Implementation of Art Therapy Expert System for Depression Using Center for Epidemiologic Studies Depression Scale." ,2016.

[7] Hyeon, J., Oh, K. J., Kim, Y. J., Chung, H., Kang, B. H., \& Choi, H. J. (2016, January). Constructing an initial knowledge base for medical domain expert system using induct RDR. In 2016 International Conference on Big Data and Smart Computing (BigComp) (pp. 408-410). IEEE.

[8] " Sensor Technologies: Healthcare, Wellness - UW Moodle Fall 2014",Michael J McGrath Cliodhna Ní Scanaill Intel Labs Europe November 2013 